\title{
Improving Construction Project Schedules before Execution
}

\author{
John Fitzsimmons ${ }^{a}$, Ying Hong ${ }^{\mathrm{b}}$ and Ioannis Brilakis ${ }^{\mathrm{b}}$ \\ ${ }^{a}$ Laing O'Rourke, United Kingdom \\ ${ }^{b}$ Department of Engineering, University of Cambridge, United Kingdom \\ E-mail:.jpf36@cam.ac.uk,yh448@cam.ac.uk, ib340@cam.ac.uk
}

\begin{abstract}
-
The construction industry has been forever blighted by delay and disruption. To address this problem, this study proposes the Fitzsimmons Method (FM method) to improve the scheduling performance of activities on the Critical Path before the project execution. The proposed FM method integrates Bayesian Networks to estimate the conditional probability of activity delay given its predecessor and Support Vector Machines to estimate the time delay. The FM method was trained on 302 completed infrastructure construction projects and validated on a $\mathfrak{\$ 4 0}$ million completed road construction project. Compared with traditional Monte Carlo Simulation results, the proposed FM method is $\mathbf{5 2 \%}$ more accurate in predicting the projects' time delay. The proposed FM method contributes to leveraging the vast quantities of data available to improve the estimation of time risk on infrastructure and construction projects.
\end{abstract}

Keywords -

Construction Schedule; Machine Learning; Project Delay; Critical Path Method

\section{Introduction}

At present, around $£ 100 \mathrm{bn}$ is spent in the UK each year on infrastructure investments [1], making the delivery of infrastructure $70 \%$ of the total spending on the National Health Service. By 2030 it is estimated that around $£ 19$ bn a year will be wasted on the avoidable costs generated by poorly delivered infrastructure projects [2]. This is a worrying trend in an age where populations are aging and productivity in the UK economy has stagnated [3]. Experts estimate that for every $£ 1$ invested in infrastructure, the benefit to the economy is $£ 2.841$ [4]; the money wasted on poorly delivered infrastructure would equate to as much as $£ 35$ bn per year in unrealised economic benefit. The cost to the global economy would be $\$ 620 \mathrm{bn}$ each year, if the trend were extrapolated worldwide [5], with around $\$ 1.1 \mathrm{tn}$ of potential loss. While delays are only part of the picture, it has been argued that they are among the most significant culprits in undermining the successful completion of infrastructure investment $[6,7]$.

Recent research used machine learning methods to predict project delay, including naïve Bayesian [8], Bayesian Belief Network [9] and logistic regression [10]. In practice, some software packages (e.g. Deltek Acumen Fuse) enable users to pre-define delay factors and its correlations while modelling risk. However, the analysis of these packages is still inarticulate and does not have a strong evidentiary basis.

This research aims to create a more objective and evidence-based framework for schedule risk analysis, given the theoretical limits and incompatible software in risk analysis. The proposed solution - the FM Method simulates risks on the critical path and predict project delay. Hence, the proposed method can provide the project manager and schedulers insights about the potential risks of their planning before execution, thus enable effective planning.

This paper starts with reviewing literature ineffective planning and project duration prediction, followed by the proposed FM method, analysis results of FM method and a discussion of analysis results. The conclusion is summarised at the end.

\section{Literature Review}

This section provides a comprehensive review in construction project delay estimation. This section starts with a review in schedule quality, followed by commonly used methods in predicting construction delay. Research gaps and questions are summarised at the end.

\subsection{Schedule Quality}

Late project delivery is affected by many factors either internally or externally, from engineering design to project management [11]. External factors including weather [12,13] and macroeconomic conditions [14] being unpredictable and uncontrollable. Whereas addressing internal factors, and in particular schedule quality [15], can significantly reduce the chance of late delivery [16]. A quality schedule uses expected outputs, 
resource and space constraints, and technical expertise to determine an optimal and achievable schedule of activities. Subjectivity and uncertainty hinder the production of quality schedules.

Schedulers estimate time-risk on construction projects and allocate time contingency for high-risk activities to minimise the possibility of project delay in common practice. However, the estimation of time-risk on construction projects is done subjectively, largely by experience, and the penetration of academic concepts into the realm of common practice is negligible [17,18]. Including time continency is one of the trickiest areas of project planning, as it deals with a level of uncertainty which can be challenging to calculate and understand without detailed knowledge of historical records [19].

\subsection{Predicting construction delay}

The most traditional way to detect project delay in the practice is the Critical Path Method (CPM) [17,20]. CPM connects a series of construction tasks with defined dependency links to create a directed acyclic graph (DAG) network. Programme Evaluation and Review Technique (PERT) is a variant of CPM that accounts uncertainty with Beta distribution [21,22]. Azaron et al. [23] look at refining the bounds of the project duration risk by introducing the concept of a dynamic Markov PERT model. The approach estimates societal factors such as war, strikes and inflation to make activity durations non-static over time. However, it is an untested model, but it begins to link external factors, deterministic CPM, PERT, and correlation together in an interesting way.

To add covariance and correlation effects to PERT and MCS, Ökmen et al. [24] construct a system called Correlated Schedule Risk Analysis (CSRAM), which uses simple subjective inputs on a range of project risks including weather, soil conditions, labour productivity, and material/resource availability factors. However, CSRAM does not address the crucial problem of subjectivity and opinion-based analysis, a factor commonly associated with disputes in contracts $[25,26]$. Furthermore, the empirical evidence that this technique is scalable and works across a range of projects types is lacking.

Recent researchers predict project delay using machine learning methods (e.g. Artificial Neural Network (ANN)) based on influencing factors (including the project manager's experience). Kog et al. [27] create a neural network for determining schedule performance from extraneous project factors, including project manager's experience and monetary incentive to the designer. Attal [28] used the overall duration and cost of highways projects to train a series of ANN to determine the key project features to be used in a duration prediction model. Hola et al. [29] take a more specific approach, using ANN to predict earthworks durations. Similarly, Bhokha et al. [30] predicted the duration of building construction using ANN.

A promising solution to the problem of co-variance and project uncertainty is the Bayesian Network (BN) approach. A BN is a probabilistic DAG network, with nodes representing outcomes and arcs, or links, representing the conditional relationships between the nodes [31]. In this respect, they are very similar to construction schedules, where each task could be considered a node and each logic link a conditional dependence relationship. $\mathrm{BN}$ has been successfully used in several fields, including medical diagnoses [32] and modelling of complex interactions including operational risk [33], environment [34], and road traffic accidents [35]. Consequently, several recent studies have proposed a framework for using $\mathrm{BNs}$ to enhance schedule risk analysis [36-38].

\subsection{Research gaps and questions}

Three research gaps are identified through extensive literature: (1) it is not known how well the subjective risk analysis studies scale up to large infrastructure projects - they either set out an untested framework or use small sample projects to demonstrate accuracy; (2) the studies which use data have done so at a macro level, which does not allow risk simulation and assessment of a baseline schedule to be undertaken; (3) natural language processing techniques have not yet been optimised for construction industry language. In essence, the research problem left unsolved is that there is no unified approach which seeks to model uncertainty in project schedules using historical data to validate all of the inputs into the process.

This study aims to answer the following research questions: (1) What techniques can be employed to prepare historical construction schedule data for prediction model training? (2) What effect will the application of these techniques, combined with prediction modelling have on the accuracy of time-risk simulation models?

\section{Proposed Method}

This section provides the proposed method. It starts with an overview of the methods. This study proposes a framework - Fitzsimmons (FM) Method for simulating schedule risk which combines the strengths of Bayesian Networks, Support Vector Machines and Monte Carlo Simulation to simulate project outcomes. Figure 1 presents an overview of the proposed FM method. This study targets an analysis risk of the critical path statically, not dynamically. Therefore, the change of critical path is not evaluated in this model. The following subsections explain the proposed FM methods 
in detail.

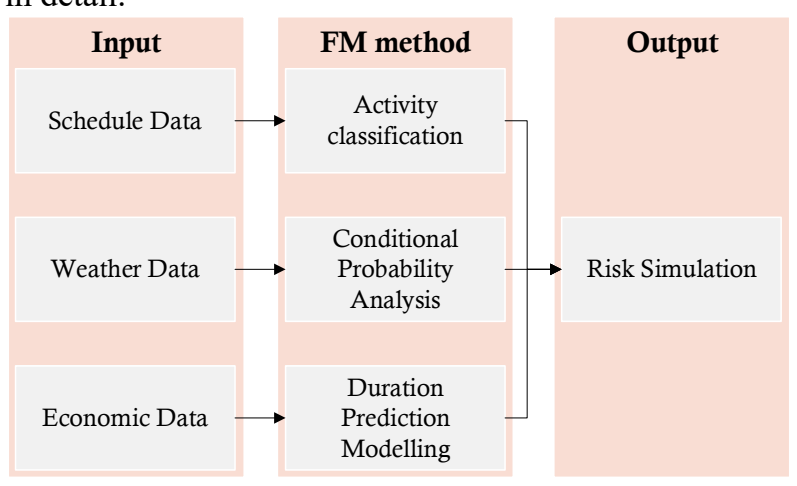

Figure 1 Overview of the proposed method

\subsection{Word Embedding}

Word embedding (Word2Vec) was used to add semantic meaning to the words in each task description. The two neural network architectures identified for this purpose were Skip-gram and Continuous bag-of-words (CBOW) embeddings. CBOW and Skip-gram are recent methods that learn word embedding representation and predict target words from context [39], but work inversely [40]. CBOW predicts target words from source text words; while, Skip-gram predicts source text words from target words [40].

It is unavoidable that there are similar activities, in terms of its functionality; for example, 'pour concrete for ring beam' and 'concrete ring beam'. Since distinguishing the semantic differences between activities remains challenging, this study clustered activities into topics using Gaussian Mixture Modelling (GMM). GMM is a probabilistic model for representing normally distributed subpopulations within an overall population [41]. The most common and well-used topic modelling methods are Latent Dirichlet Allocation (LDA) [42] and Latent Semantic Analysis (LSA) [43]. These models have been shown to work well for large corpora and documents, where keywords may appear several times [44]. Whereas, the average construction task description length is 5-10 words, rendering LDA/LSA largely ineffective. Hence, this study employed GMM to cluster construction activities. The optimal number of clusters was determined by using topic coherence as a reference. After several iterations of the test, it was difficult to determine a precise optimum number of topics.

\subsection{Bayesian Probability}

This study measures activity duration deviation, rather than activity delayed duration, to simulate the project duration. Early finished activity may also lead to project duration variation. Activity duration deviation is estimated as follows:
Duration deviation $=\left(\frac{\text { Actual duration }}{\text { original duration }}-1\right) \times 100 \%$ Eq. (1) In this study, BN is used to estimate the probability that each activity duration will deviate based on its characteristics and position within the CPM network in this study. The probability of activity duration deviation derived will be used as input in the next step. Figure 2 presents a simplified $\mathrm{BN}$ that estimates the probability of activity duration deviation.

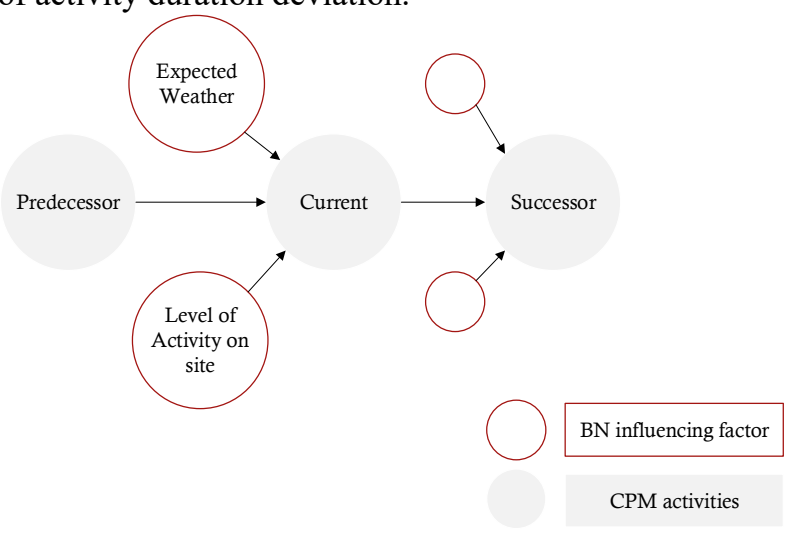

Figure 2 Simplified BN

\subsection{Support Vector Machines}

Support Vector Machines (SVM) is a classification method which distinguishes classes via solving a constrained quadratic programming problem and inserting a hyperplane [45]. SVM is used to predict the duration growth percentage of activity. Different from BN, SVM estimates the duration deviation of the current activity. Except for the probability of duration deviation derived above, SVM inputs also include the level of concurrent activity, estimated duration, and previous activity duration deviation. The dataset was split into train and test subset with a ratio of $80 / 20 \%$, to avoid overfitting, to train an accurate SVM.

SVM, in this study, predicts the duration growth percentage of activity, as highlighted earlier. A task of 200 days and a task of 2 days could both find a prediction of $400 \%$ duration increase, but with different levels of impact to project delay. Therefore, this study calibrates duration growth percentage into a relative number as compared to the activity's duration. Eq. (2) explains the duration growth percentage calibration: $\tilde{Y}=(2 \times \hat{Y} \times \delta \times \mu) /(2 \times \delta \times \mu+\hat{Y} \times \delta \times E D) E q$. (2) Where $\tilde{Y}$ is the calibrated duration growth of a given activity,

$\hat{Y}$ is the predicted duration growth of a given activity by SVM,

$\delta$ is the standard deviation of all durations in the project being simulated,

$\mu$ is the mean activity duration for the project being simulated,

$E D$ is the estimated duration of the given activity. 


\subsection{Risk simulation}

The simulation algorithm intended to calculate the average finishing position of each task over a nominal number of simulation iterations. This gives a timedistributed profile of tasks that can be used as the output of the risk analysis simulation. It is conceptually based on the Monte Carlo Simulation algorithm but with uncertainty and SVM to predict the deviation parameters. A beta distribution is used to determine the final duration deviation factor, to ensure that the duration uncertainty parameters change from one simulation iteration to the next. This distribution is parameterised by the SVM model prediction. The author chose this method to capture the strengths of MCS and PERT in modelling duration uncertainty. This model can be described as truly dynamic. With each iteration, the SVM input values will change and the duration deviation estimate will change with them, giving a broader range of possible values for the duration of each task.

\section{Data Collection and Analysis Results}

\subsection{Data Collection and Pre-processing}

The schedule data was collected from two construction and engineering firms. One of the organisations is a tier one contractor who directly delivers large infrastructure projects on behalf of public and private sector clients. The other is a schedule management consultancy that provides services to clients and contractors in several engineering disciplines. Both were requested to provide as-built schedules and initial baseline schedules for infrastructure projects regardless of the project outcome. The files were provided in the software-native Primavera P6 '.XER' format.

In total 560 project files were collected, of which 302 were valid. Some were invalid due to corrupt native files or a lack of 'actual versus planned' information. Figure 3 below shows the split by project discipline, with a relatively broad range of projects included. In total 444,173 tasks were added to the dataset, using an algorithm which interrogated the native schedule files and extracted any useful information into a large data file in '.txt' format. For context, a simple analysis of 'task count vs. project value' suggests that every 1,000 tasks represent $£ 20 \mathrm{~m}$ in project value. This indicates that the database may be equivalent to as much as $£ 8.9$ bn in completed infrastructure.

This study pre-processed activity names to secure accuracy, before feeding activity names into analysis models. Pre-processing steps include tokenisation, lemmatisation, stemming and removing stop words.
Tokenisation is a process that transforms text into tokens which are readable in a computer language [46]. Lemmatisation and stemming are used to reduce the effects of inflectional form and words' morphology [47]. Stop words (e.g. 'and', the') and punctuation was removed, to get eliminate the unmeaningful words.

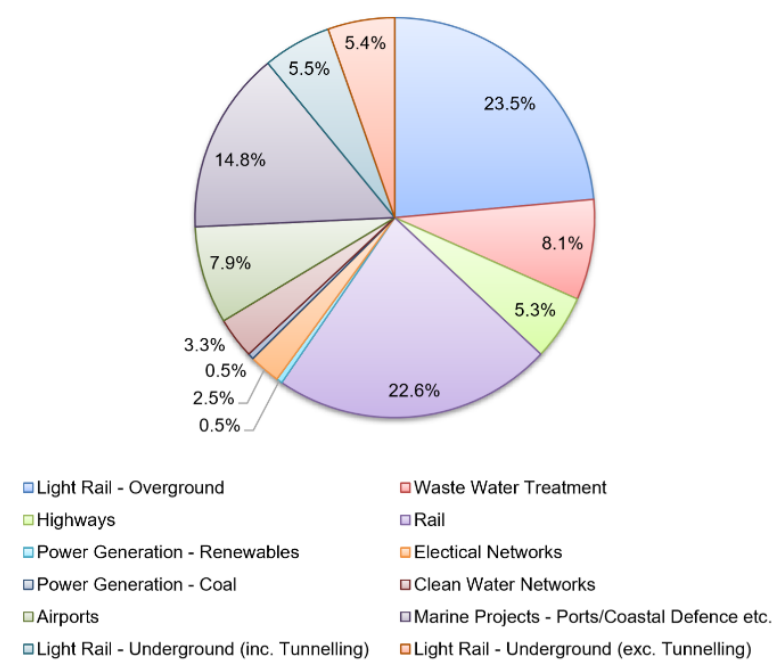

Figure 3 Data Distribution

\subsection{Analysis Results}

After pre-processing, data were fed into the proposed FM method. The following subsections summarise analysis results of each step from word embedding to simulation results.

\subsubsection{Word Embedding}

The first step in the proposed FM method represents construction activities' names with word vectors and clusters similar activities into topics. This study used Word2Vec to represent construction activities names with word vectors, as highlighted in Section 3.1. The hyperparameters of the Word $2 \mathrm{Vec}$ model are the number of training iterations, the context window length, and the vector size [48]. A range of 5, to 50 iterations was tested with no significant improvement in performance, so the default value of 5 iterations was used. The context window of 8 was selected based on the average concatenated task description and section heading length. The default vector size of 100 was used, which is suggested by literature [49,50]. In total 5,496 unique words were used in the embedding process, training across a raw dataset of 3.1 million co-occurring words.

Following that, similar activities are clustered into topics using word vectors as input. Topic modelling is an unsupervised learning method and determining the number of clusters is challenging. This study used the coherence score to determine the optimal number of 
clusters. It is found that the score settles between 10 and 25 topics, with higher scores towards 20 by plotting the coherence score. Intuitively, this feels appropriate. The design manual for roads and bridges [51] has 16 volumes; if more topics are allowed for procurement, rail and mechanical \& electrical trades, then 20 is reasonable.

\subsubsection{Activity Duration Deviation}

The SVM kernel selected was the radial basis function (RBF), which enables the SVM to perform well with high-dimensional non-linear data. The results of the activity duration growth percentage are summarised in Figure 4. The mean value of activity duration growth percentage is $114 \%$ with a standard deviation of $631 \%$. The result indicates that on average, activities are delayed for $114 \%$, as compared with its original duration.

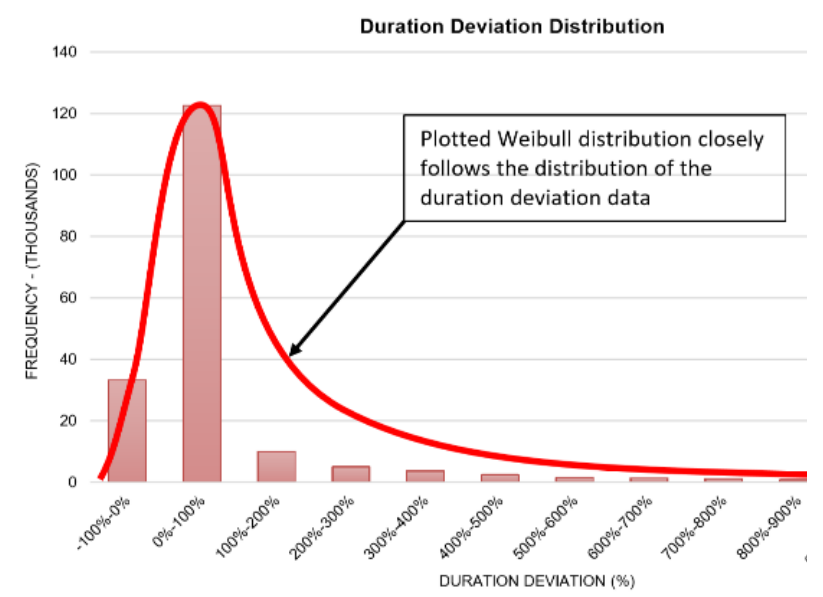

Figure 4 Distribution of Duration Deviation

Using variations about the mean is not the optimum way to simulate activity by activity variance, as shown in Figure 4. The value is heavily skewed by a few extremely high variance numbers, which in normal circumstances might be considered outliers. The issue with treating this as a normal distribution problem is that the data will never be normally distributed. Schedule duration growth works such that most samples sit around the $0 \%$ growth point - the 'quicker than planned' activities are bounded at $-100 \%$, whereas increased durations have no upper boundary. This makes deciding what constitutes an outlier a particularly difficult activity as normal conventions, involving excluding data over a certain standard deviation $(\sigma)$ threshold, cannot be meaningfully applied to datasets with large right-skewed distribution tails. The activity duration deviations appear to more closely represent a Weibull distribution as discussed by [52]. Perhaps an approach to modelling duration risk which instead selects duration deviation from a Weibull rather than a normal distribution would be a more appropriate methodology.

Significantly, the high variability was smoothed to give estimates with an average variance of around $+68 \%$ against the original duration estimate, when using SVM with a radial basis function kernel to predict the variance in an activity. The SVM results predict that some activities could finish earlier, and some activities could be late for $1,500 \%$, which is more than enough to raise a red flag to those looking for significant risks.

The accuracy of SVM results is $80 \%$ in predicting whether an activity is delayed, on time or early on the test set. The SVM model predicts a standard prediction error of $122 \%$ of activity duration. This may seem extreme, but in a very random training set with a standard deviation of $870 \%$, this is remarkably accurate.

\subsubsection{Risk Simulation}

The last step is the risk simulation. The case study used was selected randomly and excluded from model training. The selected case study is a port renovation work with a contract value at $£ 40 \mathrm{M}$ in the UK. This project finished 26 weeks later than its originally estimated duration. The key risks in this project were that the piling on the existing dock - the ground conditions and existing substructure - were unknown, as well as the condition of the existing aging structures. These were additionally subject to tidal working constraints, which exacerbated the delays experienced because of the realisation of the above risks.

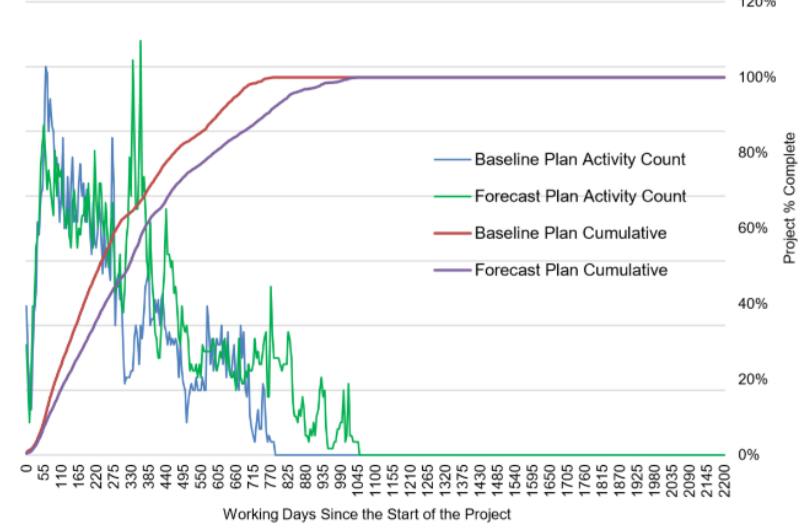

Figure 5 Baseline schedule vs FM simulation

MCS was run first, using inarticulate risk parameters derived from the literature review (best case $=90 \%$, most likely $=$ estimated duration, worst case $=130 \%$ ). Following that, the proposed FM method was run to estimate the project finishing time and compare results with the MCS results. The MCS results suggested that the project will delay for 1 week; whereas results of the FM method suggested that the project will delay for 38 weeks, which is $52 \%$ more accurate than MCS results. 
Figure 5 shows a comparison of the baseline schedule and the FM simulation over project completion. In addition, the FM method output has succeeded in assigning large time delays to the piling works and tidal interface works, so in this case study it can be considered a relative success.

\subsection{Results Interpretation}

The case studies and hypothesis test indicate that the proposed FM risk simulation methodology is significantly more accurate than the state of practice Monte Carlo Simulation. The standard prediction error was 35 weeks for MCS and 12 weeks for the FM. This suggests that the FM could be $52 \%$ more accurate than a standard MCS model. Despite these promising results, the error for the simulation of 12 weeks is still very high and the SVM model prediction standard error is also very high at $122 \%$ of the activity duration. There is ample scope to improve on the predictive accuracy of this model, although it may require a good deal more high-quality data than what has been gathered in this research.

It is also apparent from the case study that the risk simulation methodology is unable to detect some of the most significant reasons for project delay; namely: changes to scope, problems with land access, and unexpected existing site conditions. It is difficult to see from the available literature how this might change in the future, but the mitigating factor for contractors is that the time and costs associated with much of those types of delays should be recoverable through standard out-of-court contractual claim mechanisms. This would remain a highly significant problem for project promoters. The evidence is also compelling that construction activities experience delays greater than those commonly allowed for in schedule risk analysis practice.

\section{Discussion}

This study proposed an FM method which integrates BN and SVM to predict project completion time under uncertainty. This study focuses on analysing activities on the critical path to predict project duration. A domain-specific task classification model for construction schedule data has been demonstrated. This is an approach which combines unsupervised machine learning - word embedding - with Gaussian mixture models and has been adapted from methods intended to topic model social media, revealing promising if imprecise results. Bayesian Networks are used to calculate the conditional probabilities associated with the independent and duration deviation variables. Using tables of these conditional probabilities the problem is turned into a simpler Naïve Bayes or converging
Bayesian Network to calculate the probability of a task changing from its original estimated duration. MCS results suggest that the project will delay for 1 week; whereas results of FM method suggest that the project will delay for 38 weeks, which is $52 \%$ more accurate than MCS results.

In summary, there is no single technique that can be applied to the complex task of measuring risk in a noisy sequence of construction operations, using only the data contained within a Gantt chart schedule. Understanding how multiple techniques can be employed to add meaning to a construction schedule dataset, however, is a useful contribution towards leveraging the vast quantities of data available to improve the estimation of time risk on infrastructure and construction projects. Using these techniques, machines can learn about highly complex construction projects without being explicitly programmed, which presents some exciting opportunities for construction practice.

The topic modelling techniques proposed in this research could be used for a range of other purposes including automatic schedule alignment of costs and 3D objects in 5D modelling. Similar natural language techniques could also be used to monitor sentiment across a company's portfolio of projects, delivering a contemporaneous health check. Construction schedules are possibly the most up-to-date and comprehensive records on large construction projects; harnessing the value of the data they contain is a significant area of opportunity.

\section{Conclusion}

This research presents a new framework for construction schedule risk analysis. It represents the first approach to use machine learning to pre-process noisy construction schedules for a novel hybrid application of Bayesian Networks (BN) and Support Vector Machines (SVM). The proposed solution (the Fitzsimmons Method) was trained and tested on large infrastructure projects. The FM method looks at the delay risks before project execution and simulates the delay risks on the critical path. Hence, the proposed method provides the project manager and schedulers insights about the potential risks of their planning. The method is built on the work of several studies describing how machine learning can be used to approach the problem of understanding construction schedules. It has been shown that using large datasets to train prediction models can lead to superior simulation results on a limited sample, doing so with a technique eminently scalable to larger datasets and higher numbers of test projects. In tackling the problems associated with construction schedule data, some valuable contributions have been made to research around applied artificial 
intelligence. The proposed method is limited to predict the delay of critical path statically. Future research can investigate the incorporation of dynamic modelling to facilitate a real-time project delay prediction.

\section{References}

[1] HM Infrastructure and Projects Authority, National Infrastructure Delivery Plan 20162021, 2016.

[2] Mace Insights, Social Value: Underpinning our future legacy, (2017) 1-6.

[3] ONS, Construction output in Great Britain: August 2019, Off. Natl. Stat. (2019).

[4] HM Treasury, National Infrastructure Plan 2014, 2014. https://doi.org/10.4324/9781351203111-4.

[5] Oxford Economics, Global Infrastructure Outlook, 2017.

[6] B. Flyvbjerg, M. Garbuio, D. Lovallo, Delusion and deception in large infrastructure projects: two models for explaining and preventing executive disaster, Calif. Manage. Rev. 51 (2009) 170-194.

[7] F. Beckers, N. Chiara, A. Flesch, J. Maly, E. Silva, U. Stegemann, A risk-management approach to a successful infrastructure project, Mckinsey Work. Pap. Risk. (2013) 18.

[8] A. Gondia, A. Siam, W. El-Dakhakhni, A.H. Nassar, Machine Learning Algorithms for Construction Projects Delay Risk Prediction, J. Constr. Eng. Manag. 146 (2020) 4019085. https://doi.org/doi:10.1061/(ASCE)CO.19437862.0001736.

[9] V.T. Luu, S.-Y. Kim, N. Van Tuan, S.O. Ogunlana, Quantifying schedule risk in construction projects using Bayesian belief networks, Int. J. Proj. Manag. 27 (2009) 39-50. https://doi.org/https://doi.org/10.1016/j.ijproma n.2008.03.003.

[10] P.C. Anastasopoulos, S. Labi, A. Bhargava, L. Mannering Fred, Empirical Assessment of the Likelihood and Duration of Highway Project Time Delays, J. Constr. Eng. Manag. 138 (2012) 390-398.

https://doi.org/10.1061/(ASCE)CO.19437862.0000437.

[11] B. Mulholland, J. Christian, Risk Assessment in Construction Schedules, J. Constr. Eng. Manag. $125 \quad$ (1999) 8-15. https://doi.org/10.1061/(ASCE)07339364(1999)125:1(8).

[12] P.F. Kaming, P.O. Olomolaiye, G.D. Holt, F.C. Harris, Factors influencing construction time and cost overruns on high-rise projects in Indonesia, Constr. Manag. Econ. 15 (1997) 83-
94. https://doi.org/10.1080/014461997373132.

[13] O. Moselhi, D. Gong, K. El-Rayes, Estimating weather impact on the duration of construction activities, Can. J. Civ. Eng. 24 (2011) 359-366. https://doi.org/10.1139/196-122.

[14] K. Honek, E. Azar, C.C. Menassa, Recession Effects in United States Public Sector Construction Contracting: Focus on the American Recovery and Reinvestment Act of 2009, J. Manag. Eng. 28 (2012) 354-361.

[15] M.A. Bragadin, K. Kähkönen, Safety, Space and Structure Quality Requirements in Construction Scheduling, Procedia Econ. Financ. $21 \quad$ (2015) 407-414. https://doi.org/10.1016/s2212-5671(15)00193-8.

[16] M. Elzomor, R. Burke, K. Parrish, G.E. Gibson, Front-End Planning for Large and Small Infrastructure Projects: Comparison of Project Definition Rating Index Tools, J. Manag. Eng. 34 (2018).

[17] CIOB, Managing the risk of delayed completion in the 21st Century, (2009).

[18] J.I. Ortiz-González, E. Pellicer, G. Howell, Contingency management in construction projects: A survey of spanish contractors, 22nd Annu. Conf. Int. Gr. Lean Constr. Underst. Improv. Proj. Based Prod. IGLC 2014. (2014) 195-206.

[19] S.A. Mubarak, Construction project scheduling and control, John Wiley \&amp; Sons, 2015.

[20] P.D. Galloway, Survey of the construction industry relative to the use of CPM scheduling for construction projects, J. Constr. Eng. Manag. 132 (2006) 697-711.

[21] J. Fortin, P. Zieliński, D. Dubois, H. Fargier, Criticality analysis of activity networks under interval uncertainty, J. Sched. 13 (2010) 609627. https://doi.org/10.1007/s10951-010-0163-3.

[22] B. Gładysz, D. Skorupka, D. Kuchta, A. Duchaczek, Project Risk time Management - A Proposed Model and a Case Study in the Construction Industry, Procedia Comput. Sci. $64 \quad$ (2015) 24-31. https://doi.org/10.1016/j.procs.2015.08.459.

[23] A. Azaron, S.M.T. Fatemi Ghomi, Lower bound for the mean project completion time in dynamic PERT networks, Eur. J. Oper. Res. 186 (2008) 120-127. https://doi.org/10.1016/j.ejor.2007.01.015.

[24] O. Ökmen, A. Öztaş, Construction Project Network Evaluation with Correlated Schedule Risk Analysis Model, J. Constr. Eng. Manag. $134 \quad$ (2008) 49. https://doi.org/10.1061/(ASCE)07339364(2008)134:1(49). 
[25] J. Levin, Relational incentive contracts, Am. Econ. Rev. 93 (2003) 835-857.

[26] S. Mitkus, T. Mitkus, Causes of Conflicts in a Construction Industry: A Communicational Approach, Procedia - Soc. Behav. Sci. 110 (2014) 777-786. https://doi.org/10.1016/j.sbspro.2013.12.922.

[27] Y.C. Kog, D.K.H. Chua, P.K. Loh, E.J. Jaselskis, Key determinants for construction schedule performance, Int. J. Proj. Manag. 17 (1999) 351-359. https://doi.org/10.1016/S02637863(98)00058-1.

[28] A. Attal, Masters Thesis - Development of neural network models for prediction of highway construction cost and project duration, Ohio University, 2010.

[29] B. Hola, K. Schabowicz, Estimation of earthworks execution time cost by means of artificial neural networks, Autom. Constr. 19 (2010) $570-579$. https://doi.org/10.1016/j.autcon.2010.02.004.

[30] S. Bhokha, S.O. Ogunlana, Application of artificial neural network to forecast construction duration of buildings at the predesign stage, Eng. Constr. Archit. Manag. 6 (1999) 133-144.

[31] M. Scutari, Learning Bayesian networks with the bnlearn R package, ArXiv Prepr. ArXiv0908.3817. (2009).

[32] D. Nikovski, Constructing Bayesian networks for medical diagnosis from incomplete and partially correct statistics, IEEE Trans. Knowl. \&amp; Data Eng. (2000) 509-516.

[33] R.G. Cowell, R.J. Verrall, Y.K. Yoon, Modeling operational risk with Bayesian networks, J. Risk Insur. 74 (2007) 795-827.

[34] P.A. Aguilera, A. Fernández, R. Fernández, R. Rum $\backslash \backslash i$, A. Salmerón, Bayesian networks in environmental modelling, Environ. Model. \&amp; Softw. 26 (2011) 1376-1388.

[35] J. De Oña, G. López, R. Mujalli, F.J. Calvo, Analysis of traffic accidents on rural highways using Latent Class Clustering and Bayesian Networks, Accid. Anal. \&amp; Prev. 51 (2013) $1-10$.

[36] M. Fineman, Improved risk analysis for large projects: Bayesian networks approach, 2010.

[37] L. Jun-yan, Schedule Uncertainty Control: A Literature Review, Phys. Procedia. 33 (2012) 1842-1848.

https://doi.org/10.1016/j.phpro.2012.05.293.

[38] V. Khodakarami, A. Abdi, Project cost risk analysis: A Bayesian networks approach for modeling dependencies between cost items, Int. J. Proj. Manag. 32 (2014) 1233-1245. https://doi.org/10.1016/j.ijproman.2014.01.001.
[39] J. Pennington, R. Socher, C. Manning, Glove: Global vectors for word representation, in: Proc. 2014 Conf. Empir. Methods Nat. Lang. Process., 2014: pp. 1532-1543.

[40] T. Mikolov, K. Chen, G. Corrado, J. Dean, Efficient estimation of word representations in vector space, ArXiv Prepr. ArXiv1301.3781. (2013).

[41] C. Bishop, Pattern Recognition and Machine Learning, Springer-Verlag New York, 2006.

[42] D.M. Blei, A.Y. Ng, M.I. Jordan, Latent dirichlet allocation, J. Mach. Learn. Res. 3 (2003) 993-1022.

[43] S. Deerwester, S.T. Dumais, G.W. Furnas, T.K. Landauer, R. Harshman, Indexing by latent semantic analysis, J. Am. Soc. Inf. Sci. 41 (1990) 391-407.

[44] D. Mimno, H.M. Wallach, E. Talley, M. Leenders, A. McCallum, Optimizing Semantic Coherence in Topic Models, Proc. 2011 Conf. Empir. Methods Nat. Lang. Process. (2011) 262-272. https://doi.org/10.1037/1082989X.12.1.105.

[45] T. Joachims, Making large-Scale SVM Learning Practical, in: Adv. Kernel Methods - Support Vector Learn., 1999.

[46] C.D. Manning, M. Surdeanu, J. Bauer, J.R. Finkel, S. Bethard, D. McClosky, The Stanford CoreNLP natural language processing toolkit, in: Proc. 52nd Annu. Meet. Assoc. Comput. Linguist. Syst. Demonstr., 2014: pp. 55-60.

[47] N. Habash, O. Rambow, R. Roth, MADA+ TOKAN: A toolkit for Arabic tokenization, diacritization, morphological disambiguation, POS tagging, stemming and lemmatization, in: Proc. 2nd Int. Conf. Arab. Lang. Resour. Tools (MEDAR), Cairo, Egypt, 2009: p. 62.

[48] R. Řehůřek, models.word2vec - Word2vec embeddings, Gensim. (2019).

[49] R. Das, M. Zaheer, C. Dyer, Gaussian lda for topic models with word embeddings, in: Proc. 53rd Annu. Meet. Assoc. Comput. Linguist. 7th Int. Jt. Conf. Nat. Lang. Process. (Volume 1 Long Pap., 2015: pp. 795-804.

[50] V.K. Rangarajan Sridhar, Unsupervised Topic Modeling for Short Texts Using Distributed Representations of Words, (2015) 192-200. https://doi.org/10.3115/v1/w15-1526.

[51] DMRB, Design Manual for Roads and Bridges (DMRB), Standardsforhighways.Co.Uk. (2019).

[52] Y.H. Abdelkader, Evaluating project completion times when activity times are Weibull distributed, Eur. J. Oper. Res. 157 (2004) 704715 . https://doi.org/10.1016/S03772217(03)00269-8. 